\title{
The Activity and Water Footprint of Unconventional Energy Production under Hydroclimate Variation in Colorado
}

\author{
Supporting Information
}

Submitted to: ACS ES\&T Water

Xuewei Du, Huishu Li, Cristian A. Robbins, Kenneth, H. Carlson, and Tiezheng Tong*

Department of Civil and Environmental Engineering, Colorado State University, Fort Collins, Colorado 80523, United States

* Corresponding author: email: tiezheng.tong@ colostate.edu; Tel. +1 (970) 491-1913

Number of Pages: 9

Number of Tables: 4

Number of Figures: 4 


\section{Section 1. Correlation analyses of UOG activities and water footprint with hydroclimate}

condition We performed different correlation analyses to evaluate the relationship of the unconventional oil and gas (UOG) activities with hydroclimate condition and crude oil price, including both linear (Pearson correlation) and non-linear (Spearman and Kendall correlations) analyses. Specifically, Pearson's $r$ is the most commonly used correlation coefficient and measures the linear relationship between two continuous variables; ${ }^{1}$ Spearman's correlation coefficient $\left(\mathrm{r}_{\mathrm{s}}\right)$ and Kendall's coefficients $(\tau)$ assesses the monotonic relationship (whether linear or not) between two variables; ${ }^{2,3}$ For all those correlations, week or negligible relationships exists when the absolute values of correlation coefficients are less than $0.3 .^{4,5}$

As shown in Table S1, hydroclimate condition (as indicated by the drought severity classification index, DSCI) exhibited no linear relationship with the number of newly drilled wells (evidenced by Pearson's $r=0.01$ and $p>0.1$ ). Also, a positive relationship was shown by Spearman and Kendall correlations, with the correlation being weak but statistically significant $\left(\mathrm{r}_{\mathrm{s}}\right.$ and $\tau<0.3, p<0.005)$. However, the positive relationship $\left(\mathrm{r}_{\mathrm{s}}\right.$ and $\left.\tau>0\right)$ indicates that such correlations are mathematically valid but practically meaningless, because enhanced drought condition should not increase the activity of water-consumptive hydraulic fracturing in practical. In contrast, the crude oil price showed strongly positive and statistically significant relationship with the UOG activity (Table S1) in all the three analyses. Therefore, hydroclimate condition imposes a negligible impact on the drilling activity, which is mainly driven by the market.

Table S1. Correlations of DSCI and oil price with the number of newly drilled wells

\begin{tabular}{|l|l|l|l|l|l|l|}
\hline Variable & \multicolumn{4}{l}{ Raw data } & \multicolumn{4}{l|}{ Spearman } & \multicolumn{2}{l|}{ Kendall } \\
\cline { 2 - 7 } & \multicolumn{1}{|l|}{ Pearson } & $\mathbf{r}_{\mathrm{s}}$ & $\boldsymbol{p}$ & $\boldsymbol{\tau}$ & $\boldsymbol{p}$ \\
\cline { 2 - 7 } & $\mathbf{r}$ & $\boldsymbol{p}$ & 0.25 & 0.002 & 0.16 & 0.005 \\
\hline DSCI & 0.01 & 0.89 & 0.60 & $<0.001$ & 0.44 & $<0.001$ \\
\hline Oil price & 0.62 & $<0.001$ & 0.60 &
\end{tabular}


Further, hydroclimate condition, crude oil price, drilling activity, and UOG water consumption might be all subject to seasonal cycles. Therefore, we removed the seasonal components of time series in order to better understand the relationship of hydroclimate condition with activity/water consumption of UOG production. Additive decomposition of time series was performed in our analysis because the seasonal variations appears to be constant over time. ${ }^{6}$ The additive decomposition assumes that a time series is composed of three additive terms:

$$
y_{t}=S_{t}+T_{t}+R_{t}
$$

where $y_{t}$ is the raw data, $S_{t}$ is the seasonal variation, $T_{t}$ is the trend component, and $R_{t}$ is the error (or irregular component).

The decompositions of time series of DSCI, crude oil price, number of newly drilled wells, and water consumption by hydraulic fracturing $(\mathrm{HF})$ were performed using the Rstudio software in order to estimate the seasonal trend for each dataset (Figure S1). Adjusted datasets without seasonal components of time series were obtained by subtracting the seasonal trend from each dataset. Then the correlation analyses were re-calculated for the adjusted datasets and the results are shown in Table S2. 
a
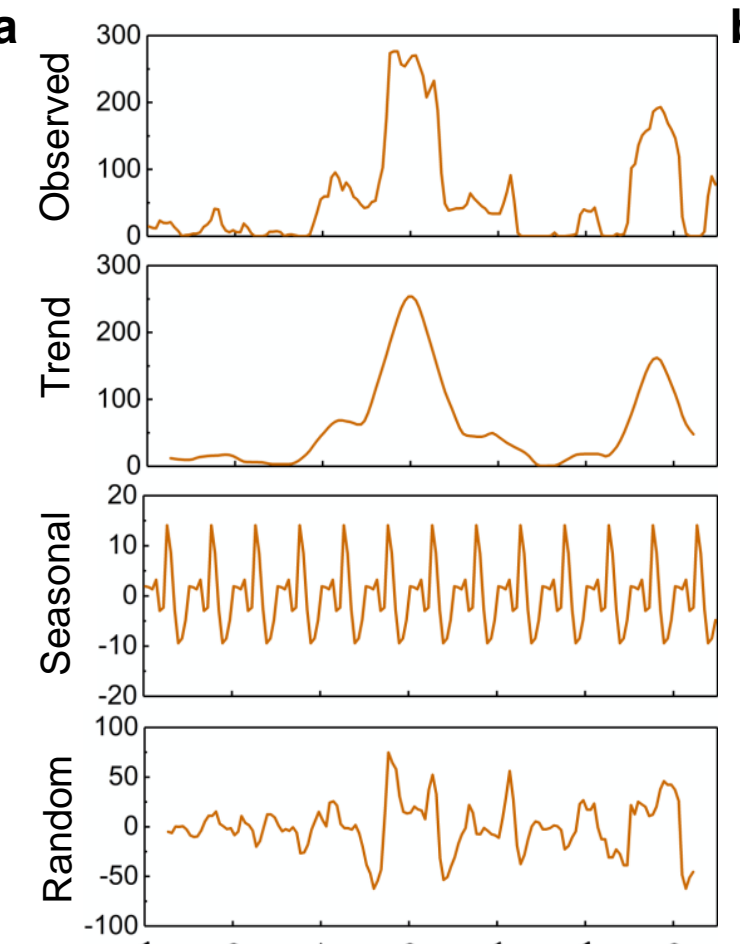

C $200^{7} 20^{09} 20^{11} 20^{13} 20^{15} 20^{17} 20^{19}$
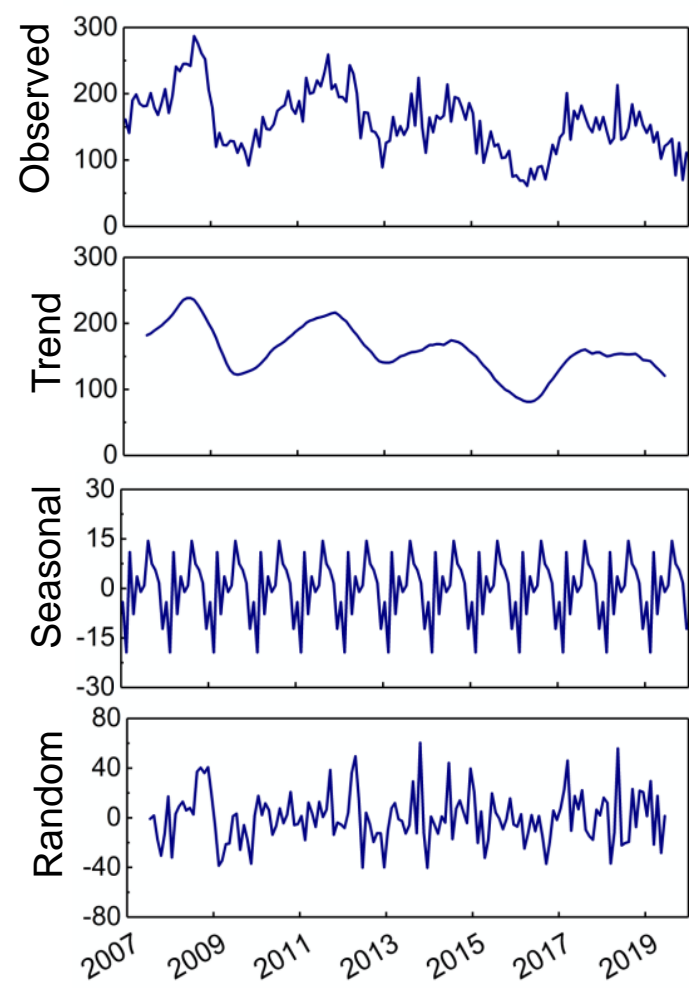
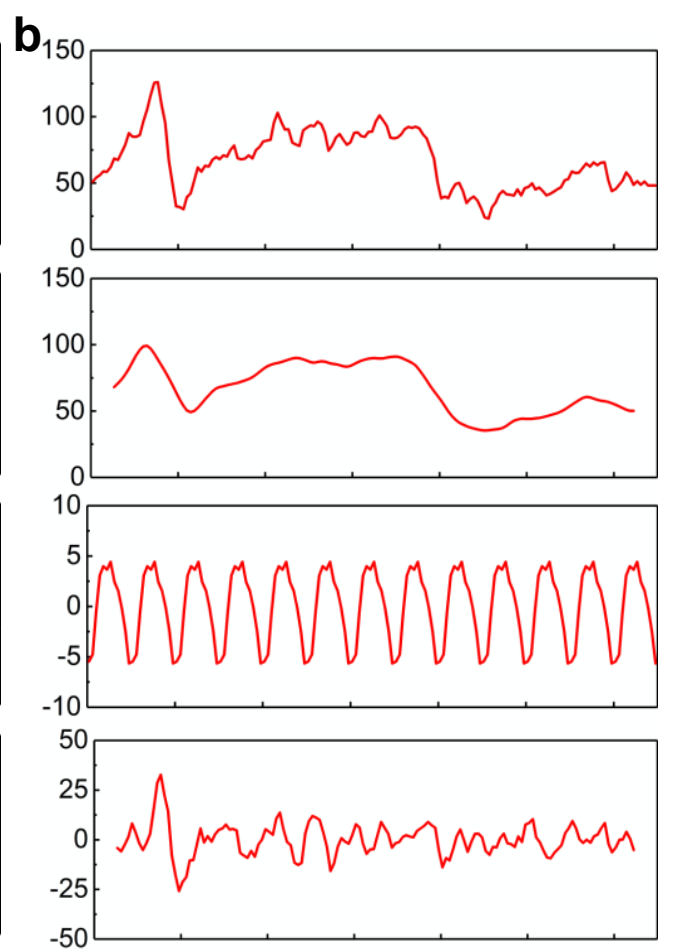

d $200^{1} \quad 20^{0^{9}} 20^{1^{1}} 20^{13} \quad 20^{15} \quad 20^{11} \quad 20^{19}$
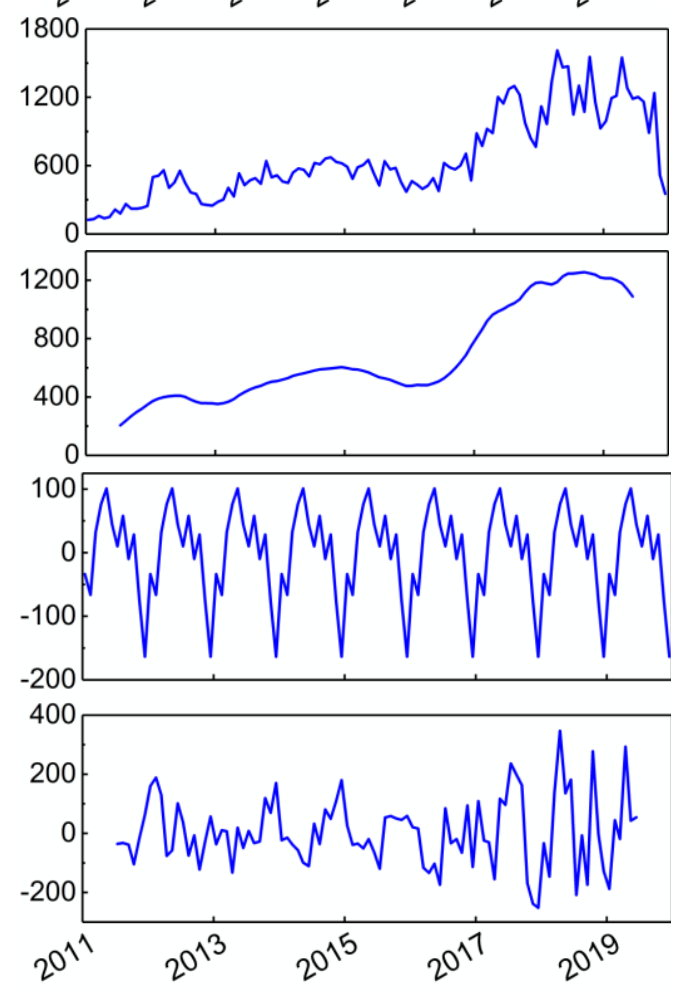

Figure S1. Decomposition of additive time series for monthly datasets of (a) DSCI, (b) crude oil price, (c) number of newly drilled wells, and (d) water consumption by HF. 
Table S2. Correlations of DSCI or oil price with the number of newly drilled wells using data after removing the seasonal trend

\begin{tabular}{|l|l|l|l|l|l|l|}
\hline Variable & \multicolumn{6}{|l|}{ Adjusted data after removing of seasonal trend } \\
\cline { 2 - 7 } & Pearson & \multicolumn{2}{l|}{ Spearman } & \multicolumn{2}{|c|}{ Kendall } \\
\cline { 2 - 7 } & $\mathbf{r}$ & $\mathbf{p}$ & $\mathbf{r}_{\mathbf{s}}$ & $\mathbf{p}$ & $\boldsymbol{\tau}$ & $\mathbf{p}$ \\
\hline DSCI & 0.01 & 0.9 & 0.21 & 0.007 & 0.14 & 0.012 \\
\hline Oil price & 0.62 & $<0.001$ & 0.61 & $<0.001$ & 0.44 & $<0.001$ \\
\hline
\end{tabular}

As shown in Table S2, the correlation results after removing the seasonal components are consistent with those obtained by the above analyses, except that the Spearman and Kendall correlations become weaker between DSCI and the drilling activity. These analyses provide additional evidence that hydroclimate variation does not affect UOG activity, although the UOG industry is water consumptive.

We also performed the correlation analyses on the relationship between hydroclimate condition and water use by HF. As shown in Table S3, the DSCI shows no linear relationship with the water consumption by HF (the absolute value of Pearson's $\mathrm{r}<0.2$, and $p=0.25$ ). However, a weakly negative monotonic correlation exists between DSCI and water consumption by HF with high significance, evidenced by both Spearman and Kendall correlations (the absolute values of $r_{\mathrm{s}}$ and $\tau<0.3, p<0.05)$. Therefore, the water consumption by HF is slightly limited under high levels of drought climates, at least mathematically. In addition, the results after decomposition of time series are consistent with the above analyses (Table S4).

Table S3 Correlation coefficients of DSCI with the water consumption by hydraulic fracturing

\begin{tabular}{|l|l|l|l|l|c|l|}
\hline Variable & \multicolumn{4}{l}{ Raw Data } & \multicolumn{4}{l|}{ Spearman } & \multicolumn{2}{l|}{ Kendall } \\
\cline { 2 - 7 } & \multicolumn{1}{|l|}{ Pearson } & $\mathbf{r}_{\text {s }}$ & $\boldsymbol{p}$ & $\boldsymbol{\tau}$ & $\boldsymbol{p}$ \\
\cline { 2 - 7 } & $\mathbf{r}$ & $\boldsymbol{p}$ & -0.23 & 0.02 & -0.16 & 0.014 \\
\hline DSCI & -0.15 & 0.25 & -0.23 & & \\
\hline
\end{tabular}

Table S4 Correlations of DSCI with the water consumption by hydraulic fracturing after removing of the seasonal trend

\begin{tabular}{|l|l|l|l|l|c|l|}
\hline Variable & \multicolumn{6}{|l|}{ After removing of seasonal trend } \\
\cline { 2 - 7 } & Pearson & \multicolumn{2}{l|}{ Spearman } & \multicolumn{2}{l|}{ Kendall } \\
\cline { 2 - 7 } & $\mathbf{r}$ & $\boldsymbol{p}$ & $\mathbf{r}_{\mathbf{s}}$ & $\boldsymbol{p}$ & $\boldsymbol{\tau}$ & $\boldsymbol{p}$ \\
\hline DSCI & -0.12 & 0.23 & -0.25 & 0.009 & -0.19 & 0.003 \\
\hline
\end{tabular}




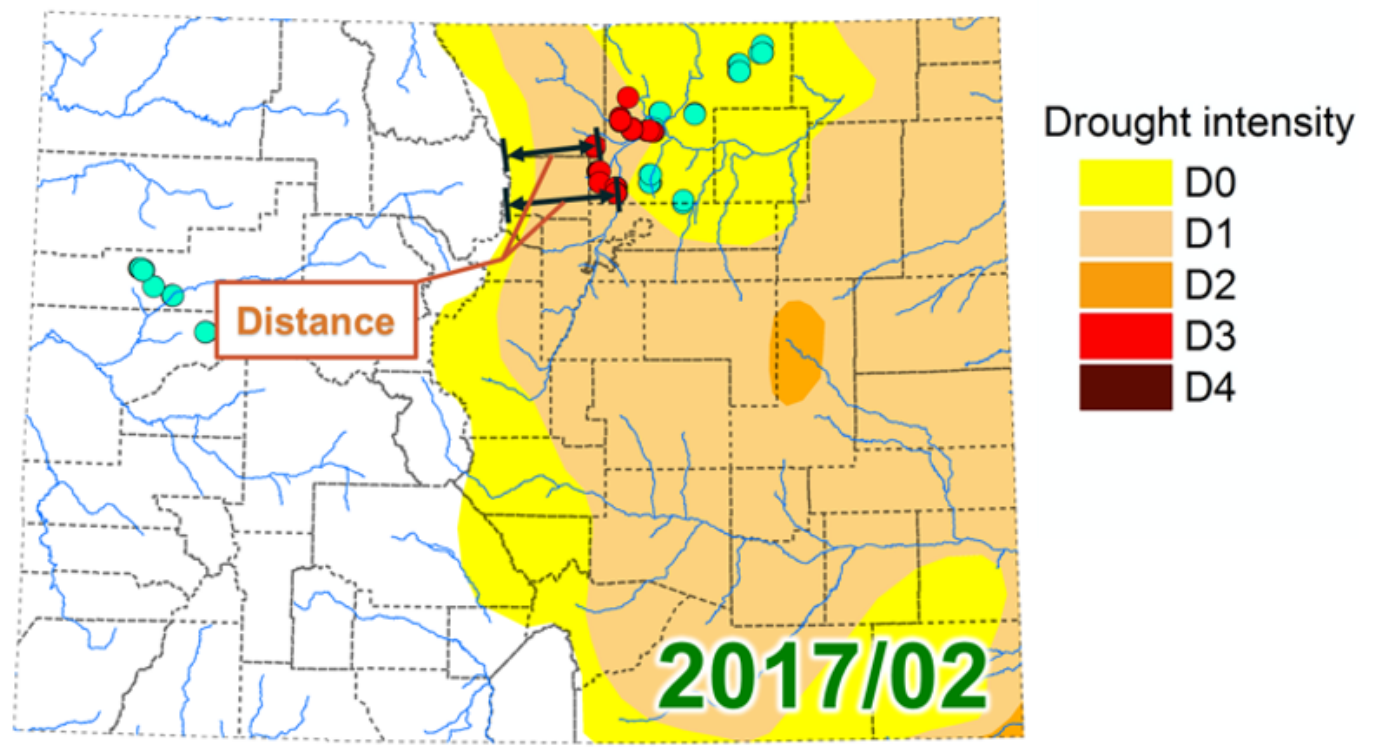

Figure S2. Schematic illustration of how the drought-escaping distance $\left(d_{e s}\right)$, which is defined as the distance between UOG wells (blue or red dots) located in drought area (D1-D4 drought categories, red dots) to the closest non-drought area (without any drought category), is calculated. Note that due to the size of the dots, one dot might cover more than one well. 


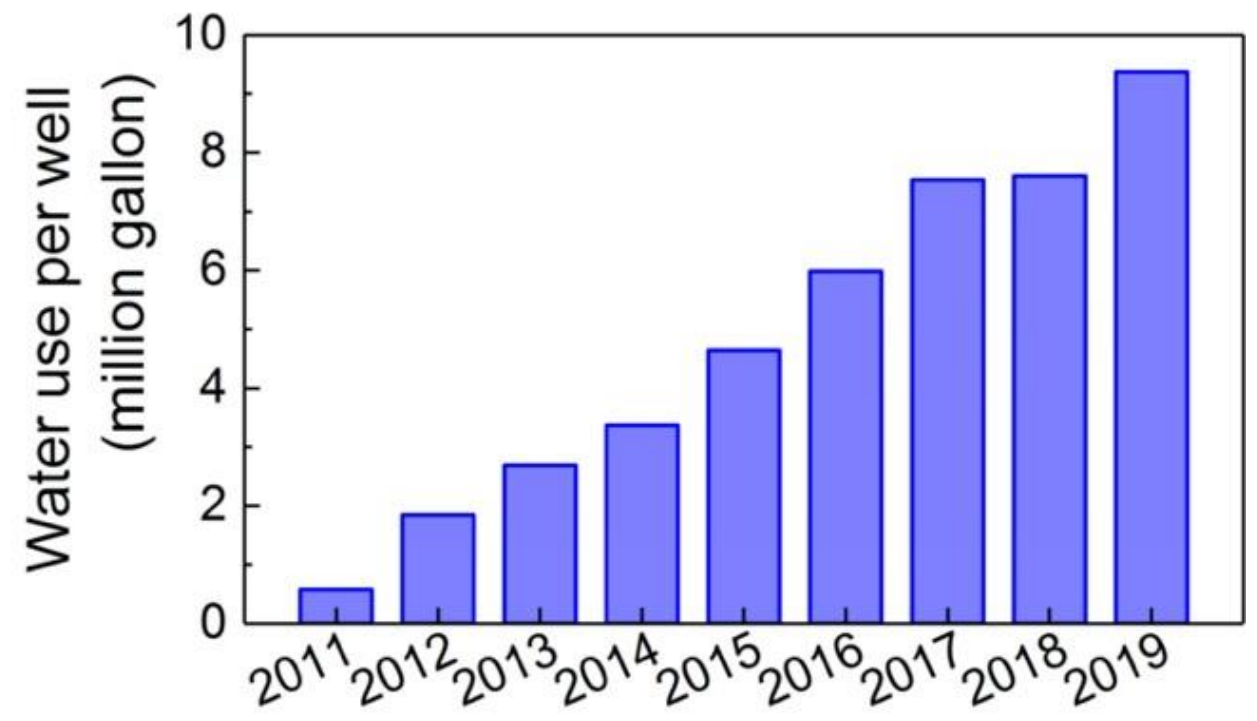

Figure S3. The annual average water use per well in Colorado between 2011 and 2019. The data are obtained from the FracFocus database. 

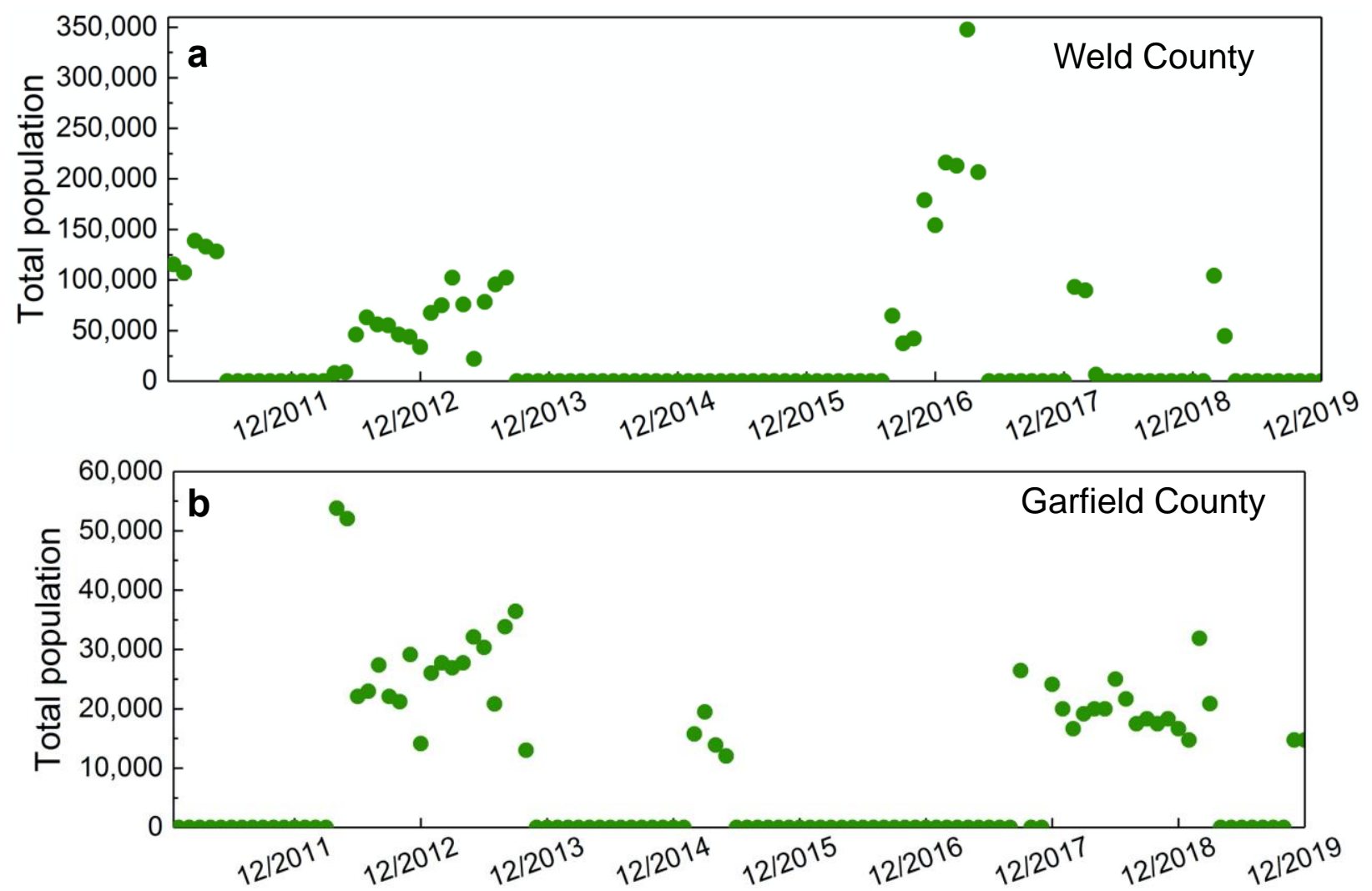

Figure S4. The population that would be sustained by water use of newly drilled UOG wells located in the drought areas (i.e., with drought categories of D1-D4) each month from 2011 to 2019 for (a) Weld County and (b) Garfield County. 


\section{References}

1. Pearson, K.; Galton, F., The life, letters and labours of Francis Galton. Cambridge University Press: Cambridge, 1914.

2. Spearman, C., The proof and measurement of association between two things. 1961.

3. Kendall, M. G., Rank correlation methods. 1948.

4. Akoglu, H., User's guide to correlation coefficients. Turkish journal of emergency medicine 2018, 18, (3), 91-93.

5. Hemphill, J. F., Interpreting the magnitudes of correlation coefficients. 2003.

6. Hyndman, R.J., \& Athanasopoulos, G. Forecasting: principles and practice, 2nd edition, OTexts: Melbourne, Australia. OTexts.com/fpp2.(Accessed on Spetember 13, 2020). 